\title{
TRANSFER OF CONTINUUM UV RADIATION INSIDE FRAGMENTED CLOUDS
}

\author{
P. BOISSE \\ Radioastronomie Millimétrique \\ E.N.S. \\ 24 Rue Lhomond \\ F-5231 Paris cedex 05 \\ France
}

\section{INTRODUCTION}

The penetration of visible and UV continuum radiation is a governing factor for many processes inside interstellar clouds. It determines for instance: 1) the overall chemical equilibrium (formation/destruction of molecules and neutral or ionized species, fractional ionization of the gas which is directly related to the coupling with the magnetic field); 2) the overall energy balance (heating of the dust, heating of the gas through collisions with electrons extracted from grains by the photoelectric effect or with grains; 3 ) cooling of the gas due to fine structure line emission (OI,CI,CII).

Ample evidence is given in these proceedings that real interstellar clouds (the giant molecular ones as well as the much more diffuse high latitude Cirrus) are very far from homogeneity. This structure must be taken into account when treating the radiation transfer problem because several orders of magnitudes differences may result.

\section{METHODS}

Analytical approach: It is a generalization of that presented in Boissé (1990)(hereafter paper I). The main assumptions are: 1) absorption and isotropic scattering by dust grains; 2) clouds are described as a statistical medium made of 2 randomly mixed discrete phases (cf. paper I for details). This model introduces as few parameters as possible: the overall cloud average opacity, the clump opacity, the clump volume filling factor, the density contrast and the dust albedo. Quantities to be determined are the average intensity at any point $\mathrm{M},<\mathrm{I}(\mathrm{M}, \theta, \varphi)>$, the mean intensity $\langle\mathrm{J}(\mathrm{M})>$ (average of $\mathrm{I}(\mathrm{M}, \theta, \varphi)$ over $\theta, \varphi)$ and the fluxes $\left\langle\Phi^{+}(\mathrm{M})\right\rangle,\left\langle\Phi^{-}(\mathrm{M})>\right.$ (along an axis normal to the assumed plane-parallel layer).

For unscattered photons an exact solution can be obtained (cf. paper I and Natta and Panagia, 1984). Including multiple scattering, a "non-local approximation" (valid at least when the clump opacity is not $>1$ ), the following system can be obtained ( $\mathrm{i}$ and $\mathrm{j}$ refering to the 2 phases; $\mathrm{z}$ is the depth in the layer of thickness L):

$$
<\mathrm{J}_{\mathrm{i}}(\mathrm{z})>=\int_{0}^{\mathrm{L}} \mathrm{F}_{\mathrm{ii}}\left(\mathrm{z}-\mathrm{z}^{\prime}\right)<\mathrm{J}_{\mathrm{i}}\left(\mathrm{z}^{\prime}\right)>\mathrm{dz} \mathrm{z}^{\prime}+\int_{0}^{\mathrm{L}} \mathrm{F}_{\mathrm{ij}}\left(\mathrm{z}-\mathrm{z}^{\prime}\right)<\mathrm{J}_{\mathrm{j}}\left(\mathrm{z}^{\prime}\right)>\mathrm{dz} \mathrm{z}^{\prime}
$$

Various types of solutions: 1) full solution of the system (obtained by iteration); 2)"effective" solution: in most cases, the full solution is very close to that for a uniform layer characterized by a modified effective extinction coefficient $\mathrm{K}_{\mathrm{e}}$ (with $\mathrm{K}_{\mathrm{e}}<<\mathrm{K}>$ ) and albedo $\omega_{\mathrm{e}}$; explicit algebraic expressions for $\mathrm{K}_{\mathrm{e}}$ and $\omega_{e}$ are available; -3$)$ approximate solution: for media with moderate average opacities one obtains an explicit form for $<\mathrm{J}(\mathrm{z})>$ by assuming only 2 or 6 discrete flight directions for photons. 
Monte-Carlo approach: the medium is a rectangular array of cells filled by material with the 2 above discrete densities. Simulations are used to validate the analytical method and estimate other parameters which cannot be computed (fluctuations ...).

\section{RESULTS}

I) Media with SAME clump and interclump dust albedo

Summary of results from paper 1: the layer behaves like a uniform one with a reduced opacity and albedo; as the medium gets more and more inhomogeneous, the observable radiation is more and more determined by the structure of the medium and less by the physical properties of the dust (cf. also Natta and Panagia, 1984 for extinction of an extended source by a non-uniform cloud).

\section{II) Media with DIFFERENT clump and interclump dust properties.}

Grain properties are likely to be density dependent (cf.Désert et al.,1990). To investigate specific effects due to different albedo values in the dense and diffuse phases, the layer to be used for comparison is the one characterized by the (uniform) mass-weighted albedo and the same density structure.

Good agreement is obtained between both methods. As expected, when material of high albedo is "hidden" in opaque clumps, the transmission factor is lower (the medium behaves like one of lower albedo).

For instance with a clump filling factor of 0.1 , a clump opacity of 2.5 , a mass fraction in the interclump phase of $37 \%$, a total average layer opacity of 10 and dust albedos of 0.7 (clumps) and 0.17 (interclump: the mass-weighted albedo is then 0.5 ) we get an effective opacity and albedo for the "equivalent uniform layer" of 6.5 and 0.29 respectively.

\section{III) Media with highly opaque clumps}

For average layer opacities larger than 10 , the validity of the assumptions required by the analytical method is questionable (cf. paper I). To discuss this point, we consider the asymptotic regime reached when the clump opacity goes to infinity (all other parameters remaining constant). We have shown that the limit given by the analytical method does exist. Both methods indicate that the asymptotic values are reached for relatively moderate clump opacities (about 6 in the case considered). For this reason, the analytical limit (although it cannot be correct) stills leads to good estimates, given the uncertainties in real cloud parameters. In such cases, an alternative approach would be to consider the transfer through a random distribution of completely opaque and partially reflecting obstacles (cf. Kamiuto, 1989).

\section{CONCLUSIONS}

We have shown that the analytical method described in paper I still leads to satisfactory results

- when both the density and the dust albedo too are subject to fluctuations,

- for large clump opacities.

From results obtained in the context of radiative transfer one may speculate that beyond this field, similar effects due to inhomogeneities will occur. Indeed, many quantities other than intensities involve a statistical average over regions characterized by a broad range of densities, temperatures, radiation fields ... These carry information not only on the physical conditions, but also (and possibly mainly !) on the relative proportion of gas in given conditions (i.e. on the scatter of physical conditions). Then, as far as the ultimate structure has not been attained, determining physical parameters from large scale data (e.g. temperatures or densities from emission line ratios as is currently done) can be very misleading.

\section{References}

Boissé, P.: 1990, Astron . Astrophys. 228, 502

Désert, F.X., Boulanger, F., Puget, J.L.: 1990, preprint

Kamiuto, K.: 1989, J. Quant. Spectrosc. Transfer 41, 23

Natta, A. and Panagia, N.: 1984, Astrophys. J. 287, 228 